\title{
ECG Imaging of Focal Atrial Excitation: Evaluation in a Realistic Simulation Setup
}

\author{
Danila Potyagaylo ${ }^{1}$, Axel Loewe ${ }^{1}$, Peter van Dam² ${ }^{2}$ Olaf Dössel ${ }^{1}$ \\ ${ }^{1}$ Institute of Biomedical Engineering, Karlsruhe Institute of Technology (KIT), Karlsruhe, Germany \\ ${ }^{2}$ Donders Institute for Brain Cognition Behaviour, Radboud University Medical Center, Nijmegen, \\ The Netherlands
}

\begin{abstract}
One promising application of electrocardiographic (ECG) imaging is noninvasive reconstruction of atrial activities. However, despite numerous clinical studies, which are mostly concerned with complex irregular excitation patterns, there are relatively few in silico investigations on the imaging of ectopic activation. In the present work, we explore the localization accuracy of ECG imaging regarding atrial focal sites. For the forward calculations, we used four realistic geometrical models with complex anatomical structure and a rule-based fiber orientation embedded into the atrial model. Excitation propagation was simulated with the monodomain model. For each geometrical model, 20 activation sequences originating from the posterior wall of the left atrium were simulated. Based on the bidomain theory, the body surface potential maps resulting from these focal events were computed. For the inverse reconstructions, we employed a full-search procedure based on the fastest route algorithm assuming uniform excitation propagation. Localization errors were revealed to be dependent on the model-specific atrial geometry. We also performed similarity analysis for the first halves of the $P$ wave duration, which improved the results in three models.
\end{abstract}

\section{Introduction}

Electrocardiographic imaging, or inverse problem of electrocardiography (ECG), is an evolving technology for noninvasive reconstruction of electrical cardiac activity from multichannel ECG recordings. Recently, the ECG imaging field has been experiencing great attention from both clinical and engineering communities facilitating validation of this mathematical technique in human experiments. From the perspective of possible clinical impact, noninvasive imaging of atrial arrhythmias can significantly contribute to standard pre-interventioanl diagnostic and therapy planning for patients with abnormal heart rhythm.
Though there are multiple studies addressing the quality of inverse reconstructions in atria, the majority of investigations published recently are concerned with complex irregular arrhythmias: atrial fibrillation and atrial flutter $[1,2]$. Concerning application of the noninvasive imaging to arrhythmias of focal nature, clinical cases for premature atrial complex and atrial tachycardia are described in [3]. Feasibility studies for activation times (AT) imaging of paced atrial sequences are presented in [4-6]. The work that is methodologically closest to the one presented hereafter estimated the timing of one normal atrial depolarization as a proof-of-concept for the fastest route algorithm based on the intra-myocardial distance function [7].

Despite this variety of clinical reports, there are only few simulation studies targeting the inverse problem of ECG for atria (e.g. [8,9]). Motivated by these observations, in the present work we assess the performance of AT imaging with the fastest route algorithm for localization of ectopic foci in silico for the cohort of four torso models.

\section{Methods}

\subsection{Anatomical models}

MRI data of four healthy male subjects were acquired as described in $[10,11]$. The atria and thoraces were manually segmented and combined to a voxel format, which was furthermore converted to a tetrahedral mesh for the calculation of electric potentials. The voxel resolution for electrophysiological simulations in the atria was $0.33 \mathrm{~mm}$. In the tetrahedral meshes, the mean node distance between atrial nodes was $\approx 1 \mathrm{~mm}$, which resulted in the total number of nodes between 0.35 and 0.49 million for the four models. The tetrahedral geometries contained atrial and ventricular blood, lungs, liver, spleen, spinal cord and intestines as inhomogeneous compartments.

For simulation of anisotropic excitation propagation in the atria, their voxel models were enhanced by the following structures: Crista Terminalis, pectinate muscles, Bachmanns Bundle (BB), inferior isthmus, atrio-ventricular 
rings and atrial appendages. These anatomical parts along with the corresponding fiber orientation were annotated using the rule-based algorithms described in [12]. Left-toright atrial conducting pathways included $\mathrm{BB}, 2$ posterior interatrial connections and the coronary sinus $[10,11]$.

\subsection{Electrophysiological models}

For the representation of ion kinetics at each atrial voxel a heterogeneous version of the Courtemanche model was used [13]. The ratios between conductivities in the longitudinal and transversal directions for atrial tissue, Crista Terminalis, pectinate muscles and $\mathrm{BB}$ were set to 3.75 , $6.56,10.52$ and 9, respectively. The monodomain solver acCELLerate was employed for computation of excitation propagation [10]. For each geometrical model, 20 ectopic sequences originating from the left atrium (LA) were simulated.

\subsection{Forward calculations}

From the transmembrane voltages (TMV) $V_{m}$ the extracellular electrical potentials $\phi$ were obtained by solving the following bidomain equation with the finite element method:

$$
\nabla \cdot\left(\left(\sigma_{e}+\sigma_{i}\right) \nabla \phi\right)=-\nabla \cdot\left(\sigma_{i} \nabla V_{m}\right)
$$

where $\sigma_{e}$ and $\sigma_{i}$ are electrical conductivities in the extraand intracellular spaces. The conductivity values for inhomogeneous regions within the torso were assigned as in [11]. After solving the forward problem, potential values at 120 positions uniformly distributed over the thoraces were taken as the input ECG for the inverse calculations.

\subsection{Inverse calculations}

A transfer matrix connecting the sources TMV to the ECG was calculated with the boundary element method. For that purpose, triangulated surfaces of the original torso and atrial meshes were extracted first. Afterwards, the number of nodes was reduced by edge collapsing, which resulted in an average of 2,000 and 2,800 nodes for torso and atria, respectively. Therewith, a homogeneous volume conductor model was used for subsequent full-search procedure based on the fastest route algorithm (FRA) [7].

\subsection{Fastest route algorithm}

Within the FRA-formulation, each cardiac node is assumed to be a potential excitation origin [7]. An activation sequence starting from this point is calculated with Dijkstra's algorithm and then converted to a body surface potential map (BSPM) based on the corresponding transfer

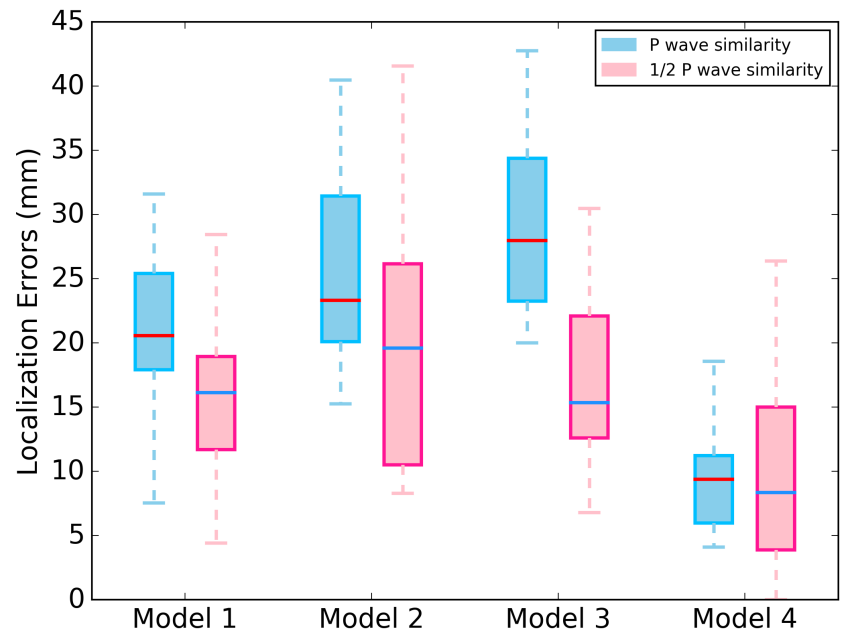

Figure 1: Localization errors obtained by FRA based on the $\mathrm{P}$ wave similarity and $1 / 2 \mathrm{P}$ wave similarity analysis: Each box plot represents errors for 20 focal activation sequences in the LA.

matrix. The node delivering the highest correlation coefficient (CC) between the measured and simulated ECG is taken to be the excitation origin. Moreover, during this procedure, CC map is generated, which can be regarded as an uncertainty quantification tool highlighting the areas of most probable location for an ectopic focus.

Within FRA, the timing matrix is dependent on the assigned conduction velocities (CVs) between each pair of nodes. For this work, we calculated the timing matrices based on the assumption of uniform propagation.

In the inverse calculations with the FRA, the duration of a test activation sequence is scaled to match the measured signals. Afterwards, the correlation is computed between the BSPMs corresponding to the input and output $\mathrm{P}$ waves. In $[10,11]$, the contribution of the right atrium (RA) to the $\mathrm{P}$ wave was shown to be dominant over those from the LA. As a uniform CV was assumed for all atrial nodes in the inverse model, we conjectured that the difference in timings of crossing a conduction bridge from the LA to the RA between the true and test activation sequences might have a great impact on the resulting correlation map. With these considerations in mind, we aimed at separation of left and right atrial contributions to the ECG by computing the correlation only between the first halves of the $\mathrm{P}$ waves duration, 1/2 $\mathrm{P}$ wave similarity in the following.

\section{Results}

For all models and ectopic activation sequences, we performed FRA analysis based on the complete and 1/2 P waves morphologies and calculated distances between the 
simulated origins and nodes exhibiting the highest correlation. The resulting localization errors are provided in Fig. 1. First of all, for the standard FRA approach, we observed similar localization errors for the models 1 to 3 and significantly better performance for model 4 . Considering only the first half of the $\mathrm{P}$ wave for correlation analysis resulted in overall (interquartile range and median) improvement of distance errors for models 1 to 3 . In contrast to that, for model 4 the errors slightly increased.

In Fig. 2 some exemplary activation sequences and CC maps for model 1 are shown. For the focal source that resulted in the largest FRA-based localization error, the ground-truth and the reconstructed AT patterns were similar in the LA but differed in the RA. While the monodomain-simulated excitation spread from the coronary sinus in the direction of the vena cava inferior, the FRA-modeled AT featured frontal propagation from the $\mathrm{BB}$ towards the RA anterior wall due to the isotropy assumption. For this case, $1 / 2 \mathrm{P}$ wave analysis was able to reduce the errors caused by assuming a wrong connection between the LA and RA and decreased the localization error from $31.6 \mathrm{~mm}$ to $18.3 \mathrm{~mm}$.

Furthermore, the ectopic foci localization errors were interpolated onto the atrial surface in order to estimate the areas most suffering from the assumption of a uniform propagation in the inverse modeling. As seen from Fig. 2, the left superior pulmonary vein was susceptible with respect to the errors made by FRA in model 1 .

\section{Discussion and conclusions}

In this work, we investigated the performance of the FRA-based full-search for localization of ectopic foci in a cohort of four highly realistic torso models in combination with a state-of-the-art electrophysiological model of the human atria. Furthermore, we showed that using only the first half of the $\mathrm{P}$ wave improves the localization of ectopic activation originating from the LA.

For all models, we interpolated errors stemming from 20 inverse reconstructions onto the atrial surface and analysed the resulting error distributions. Unfortunately, in each model, peaks of localization inaccuracies up to $4 \mathrm{~cm}$ were observed in clinically relevant areas around the pulmonary veins (PVs). Nevertheless, in most of the cases, the correlation analysis was able to correctly identify the target PV but located the focus on its wrong side (as e.g. in Fig. 2).

With respect to the $1 / 2 \mathrm{P}$ wave similarity analysis, we hypothesized that once the excitation wavefront originating from the LA has passed a conduction bridge to the RA, the contribution of the RA to the $\mathrm{P}$ wave could become dominant and strongly affect performance of the FRAbased ECG imaging. Restriction of the ECG signal to its first half improved the localization errors and featured more compact correlation maps for models 1 to 3. Despite an overall superior performance compared to the standard FRA approach, the $1 / 2 \mathrm{P}$ wave similarity criterion also resulted in the largest distance errors around the PVs. We plan to investigate this phenomena in our future work.

Within the considered cohort, we observed deviating behaviour of the FRA for model 4. In contrast to the other models, the standard formulation resulted in markedly lower localization errors and the $1 / 2 \mathrm{P}$ wave approach slightly worsened the estimation accuracy. These phenomena might be explained by the fact that the geometrical atrial model 4 was considerably smaller than other models, which was reflected in the $\mathrm{P}$ waves shortened by 10 to $30 \mathrm{~ms}$ with respect to models 1 to 3 . As a consequence, precise identification of a correct pathway between the atria was less important for the correlation-based FRA.

Having summarized our findings, we would like to provide some guidelines for improved ECG imaging in combination with the fastest route algorithm. First, a precise anatomical model of both atria is of crucial importance for any model-based approaches. Second, one could introduce a rule-based anisotropy architecture into the inverse model and assign the conduction velocities in a heterogeneous way accordingly to the available models. Finally, as we showed in the present work, it can be advisable to perform the $1 / 2 \mathrm{P}$ wave similarity analysis and compare the generated correlation maps: from our experience, better model assumptions result in more compact correlation maps with higher CC values.

In order to strengthen these statements, the study should be extended to an analysis of clinically recorded data. In our future work, we intend to add some modeling errors in the BEM: scale the atria to mimic contraction and move it by one or $2 \mathrm{~cm}$ in the vertical (with respect to torso axis) direction. As the atria move a lot during the cardiac cycle, this might provide us an idea of the required model accuracy. Furthermore, it would be very interesting to compare the obtained results with the established potential-based inverse methods that do not impose any propagation scheme and solve instead the corresponding optimization problems.

\section{References}

[1] Haissaguerre M, Shah AJ, Cochet H, Hocini M, Dubois R, Efimov I, Vigmond E, Bernus O, Trayanova N. Intermittent drivers anchoring to structural heterogeneities as a major pathophysiological mechanism of human persistent atrial fibrillation. J Physiol 2016;594(9):2387-2398.

[2] Pedrón-Torrecilla J, Rodrigo M, Climent A, Liberos A, Pérez-David E, Bermejo J, Arenal A, Millet J, FernándezAvilés F, Berenfeld O, et al. Noninvasive estimation of epicardial dominant high-frequency regions during atrial fibrillation. J Cardiovasc Electrophysiol 2016;

[3] Shah AJ, Lim HS, Yamashita S, Zellerhoff S, Berte B, Mahida S, Hooks D, Aljefairi N, Derval N, Denis A, Sacher 

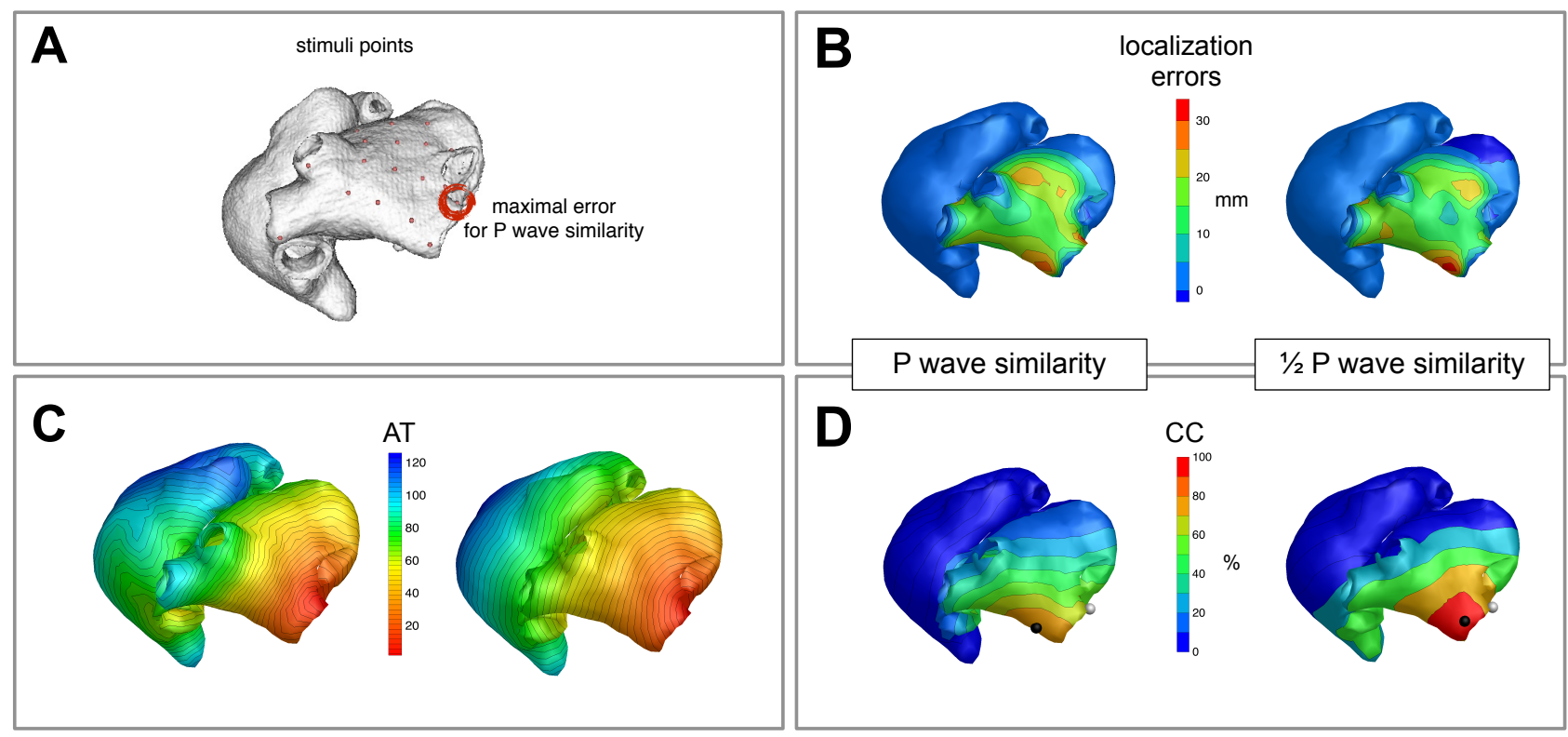

Figure 2: Model 1: A: the excitation origins under investigation are marked in red with the point resulting in the largest localization error being highlighted. B: localization distance errors interpolated on the atrial surface. C: monodomainsimulated AT versus the FRA activation sequence starting from the same point. D: CC maps for the case that resulted in the largest localization error with the standard FRA search.

F, Jais P, Dubois R, Hocini M, Haissaguerre M. NonInvasive ECG mapping to guide catheter ablation. J Atr Fibrillation 2014;7(3):31-38.

[4] Hanser F, Tilg B, Modre R, Fischer G, Messnarz B, Hintringer F, Roithinger FX. Feasibility of atrial activation time imaging. In Comput Cardiol, volume 29. 2002; 601-604.

[5] Tilg B, Fischer G, Modre R, Hanser F, Messnarz B, Schocke $M$, Kremser C, Berger T, Hintringer F, Roithinger FX. Model-based imaging of cardiac electrical excitation in humans. IEEE Trans Med Imag 2002;21(9):1031-1039.

[6] Modre R, Tilg B, Fischer G, Hanser F, Messnarz B, Seger M, Schocke MFH, Berger T, Hintringer F, Roithinger FX. Atrial noninvasive activation mapping of paced rhythm data. J Cardiovasc Electrophysiol 2003;14(7):712-719.

[7] Oosterom van A, van Dam PM. The intra-myocardial distance function as used in the inverse computation of the timing of depolarization and repolarization. In Comput Cardiol. 2005;

[8] Rodrigo M, Guillem MS, Climent AM, Pedron-Torrecilla J, Liberos A, Millet J, Fernandez-Aviles F, Atienza F, Berenfeld O. Body surface localization of left and right atrial high-frequency rotors in atrial fibrillation patients: a clinical-computational study. Heart Rhythm 2014; 11(9):1584-1591.

[9] Alday EAP, Colman MA, Langley P, Butters TD, Higham J, Workman AJ, Hancox JC, Zhang H. A new algorithm to diagnose atrial ectopic origin from multi lead ECG systems- insights from 3D virtual human atria and torso. PLoS Comput Biol 2015;11(1):e1004026.

[10] Loewe A, Krueger MW, Platonov PG, Holmqvist F, Dössel O, Seemann G. Left and right atrial contribution to the $\mathrm{P}$-wave in realistic computational models. In Functional Imaging and Modeling of the Heart 2015, Lect Notes Comput Sci, number 9126. 2015; 439-447.

[11] Loewe A, Krueger MW, Holmqvist F, Dössel O, Seemann $\mathrm{G}$, Platonov PG. Influence of the earliest right atrial activation site and its proximity to interatrial connections on $\mathrm{p}$ wave morphology during sinus rhythm. Europace ;In press.

[12] Wachter A, Loewe A, Krueger MW, Dössel O, Seemann G. Mesh structure-independent modeling of patient-specific atrial fiber orientation. In Current Directions in Biomedical Engineering, volume 1. De Gruyter, 2015; 409-412.

[13] Krueger MW, Dorn A, Keller DUJ, Holmqvist F, Carlson J, Platonov PG, Rhode KS, Razavi R, Seemann G, Dössel O. In-silico modeling of atrial repolarization in normal and atrial fibrillation remodeled state. Med Biol Eng Comput 2013;51(10):1105-1119.

Address for correspondence:

Danila Potyagaylo

Institute of Biomedical Engineering, KIT,

Fritz-Haber-Weg 1, 76131 Karlsruhe, Germany publications@ibt.kit.edu 